Фомин Дмитрий Владимирович

Амурский государственный университет,

г. Благовещенск, Россия

E-mail: e-office@yandex.ru

Fomin Dmitriy Vladimirovich

Amur State University,

Blagoveshchensk, Russia

E-mail: e-office@yandex.ru

Новгородцев Никита Сергеевич

Амурский государственный университет,

г. Благовещенск, Россия

E-mail: nnc19991999@gmail.com

Novgorodtsev Nikita Sergeevich

Amur State University,

Blagoveshchensk, Russia

E-mail:nnc19991999@gmail.com

Струков Дмитрий Олегович

Амурский государственный университет,

г. Благовещенск, Россия

E-mail: tokloo@yandex.ru

Strukov Dmitriy Olegovich

Amur State University,

Blagoveshchensk, Russia

E-mail: tokloo@yandex.ru

Поляков Алексей Вячеславович

Амурский государственный университет,

г. Благовещенск, Россия

E-mail: polyakov_a_1999@mail.ru

Polyakov Alexey Vyacheslavovich

Amur State University,

Blagoveshchensk, Russia

E-mail:polyakov_a_1999@mail.ru

ФОРМИРОВАНИЕ ТОНКИХ ПЛЕНОК $\mathrm{Mg}_{2} \mathrm{Si} \mathrm{HA} \mathrm{Si} \mathrm{(111)}$

И ИССЛЕДОВАНИЕ ИХ МЕТОДОМ ЭОС И СХПЭЭ

\title{
FORMATION OF $\mathrm{Mg}_{2} \mathrm{Si}$ THIN FILMS ON Si (111) AND THEIR RESEARCH BY EOS AND EELS
}

Аннотация В работе представлена информация о результатах формирования тонкой пленки $\mathrm{Mg}_{2} \mathrm{Si}$ на кремниевой подложке методом твердофазной эпитаксии в сверхвысоковакуумной камере прибора РНI model 590. Существует ряд трудностей при формировании пленок силицида магния из-за низкого коэффициента конденсации и высокого давления его паров. В настоящее время ведется поиск эффективных методов формирования $\mathrm{Mg}_{2} \mathrm{Si}$. В результате про- 
веденного нами эксперимента была получена тонкая пленка, исследованная in-situ методами электронной оже-спектроскопии и спектроскопии характеристических потерь энергии электронами. Анализ спектра оже-электронов показал наличие атомов магния и кремния в составе сформированной пленки. Из анализа спектров ХПЭЭ было установлено, что сформирована тонкая пленка силицида магния.

Abstract. The paper presents information on the results of the formation of a thin $\mathrm{Mg}_{2} \mathrm{Si}$ film on a silicon substrate by solid-phase epitaxy in an ultrahigh-vacuum chamber of the PHI model 590 device. There are a number of difficulties in the formation of magnesium silicide films due to the low condensation coefficient and high vapor pressure. Effective methods for the formation of $\mathrm{Mg}_{2}$ Si are currently being sought. As a result of our experiment, a thin film was obtained, which was studied in-situ by the method of electron Auger spectroscopy and spectroscopy of characteristic energy losses by electrons. Analysis of the Auger electron spectrum showed the presence of magnesium and silicon atoms in the composition of the formed film. From the analysis of the EELS spectra, it was found that a thin film of silicide magnesium was formed.

Ключевые слова: кремний, магний, тонкие пленки, твердофазная эпитаксия, силицид магния, метод электронной оже-спектроскопии, спектроскопия характеристических потерь энергии электронами

Key words: silicon, magnesium, thin films, solid-phase epitaxy, magnesium silicide, electron Auger spectroscopy, electron energy loss spectroscopy.

DOI: 10.22250/jasu.93.6

Исследование $\mathrm{Mg}_{2} \mathrm{Si}$ в его объемном состоянии позволило установить, что данный силицид является узкозонным полупроводником со значениями запрещенной зоны, лежащими в диапазоне 0,6-0,8 эВ, обладающий фотопроводимостью, которая позволяет рассматривать $\mathrm{Mg}_{2} \mathrm{Si}$ как перспективный материал для оптоэлектроники [1]. Исследование его фотоэлектрических свойств показало, что фотоотклик наблюдается до длин волн 1800-2100нм [2], а фоточувствительность при 1310 нм достигает нескольких десятков мА/Вт при малом обратном смещении [3]. Не менее привлекательным использование $\mathrm{Mg}_{2} \mathrm{Si}$ видится при создании термоэлектрических преобразователей [4]. Ожидание прогресса в формировании и исследовании свойств $\mathrm{Mg}_{2} \mathrm{Si}$ связывают с получением тонких пленок этого силицида. Однако при формировании этих пленок существует ряд трудностей из-за низкого коэффициента конденсации и высокого давления паров $\mathrm{Mg}_{2} \mathrm{Si}$ [1]. Поэтому используют различные методы получения пленок $\mathrm{Mg}_{2} \mathrm{Si}$ [1,5,6,7 и др.]. Например, в работе [1] описывается совместное осаждение магния и кремния со скоростью осаждения магния в 4-10 раз большей, чем у кремния, а температура подложки в процессе роста не превышает $200{ }^{\circ} \mathrm{C}$. В работе [5] пленки $\mathrm{Mg}_{2} \mathrm{Si}$ формировали послойно на затравочный слой магния с последующим его отжигом при температуре $380^{\circ} \mathrm{C}$.

\section{Эксперимент}

Формирование тонких пленок $\mathrm{Mg}_{2} \mathrm{Si}$ с недавнего времени стали осуществлять в лаборатории физики поверхности НОЦ АмГУ, имеющей опыт формирования пленок других полупроводниковых силицидов - $\mathrm{BaSi}_{2}$ и $\mathrm{Ca}_{2} \mathrm{Si}$ на $\mathrm{Si}(111)$ [8-10 и др.]. При этом для получения тонких пленок $\mathrm{Mg}_{2} \mathrm{Si}$ предложено использовать метод твердофазной эпитаксии, реализованный в сверхвысоковакуумной камере прибора PHI model 590 с базовым давлением $1 \cdot 10^{-9}$ Торр. Осаждение магния в нашем случае выполнялось из термоисточника, изготовленного из танталовой фольги в виде трубочки с проколом, а кремния - посредством прогрева пластины, вырезанной из промышленной шайбы FZN-100 Si(111). Подложка для образцов изготавливалась из шайбы КЭФ-100 $\mathrm{Si}(111)$ с удельным сопротивлением 
2-15 Ом*см. Источник и подложка из кремния подвергались очистке по стандартам микроэлектронного производства, включающим очистку от органических элементов и ионную очистку в химических растворах (деионизированная вода, гидрат аммиака, пероксид водорода и деионизированная вода, соляная кислота, пероксид водорода) при температуре $80^{\circ} \mathrm{C}$ в течение 10 мин., а также подвергались прогреву в течение 1,5 час. при температуре $600^{\circ} \mathrm{C}$ и кратковременным высокотемпературным прогревам при $\mathrm{T}=1250^{\circ} \mathrm{C}$. Скорость осаждения калибровалась с использованием кварцевого датчика, подключенного к прибору Sycon Instruments, и составила 1,5 нм/мин для магния и 0,2 нм/мин для кремния. Сначала на подготовленном образце был сформирован буферный слой кремния толщиной 60 нм, а затем затравочный слой магния толщиной 1,5 нм при температуре $100^{\circ} \mathrm{C}$. Далее было проведено соосаждение $\mathrm{Mg}$ и Si c соотношением скоростей 7:1, в результате была сформирована тонкая пленка силицида магния толщиной 100 нм. Далее in-situ проводилось ее исследование методом оже-электронной спектроскопии (ЭОС) и спектроскопии характеристических потерь энергии электронами (СХПЭЭ)

\section{Результаты и их обсуждение}

Спектры оже-электронов, полученные при формировании тонкой пленки силицида магния, представлены на рис. 1. График содержит спектры оже-электронов, полученные от буферного слоя

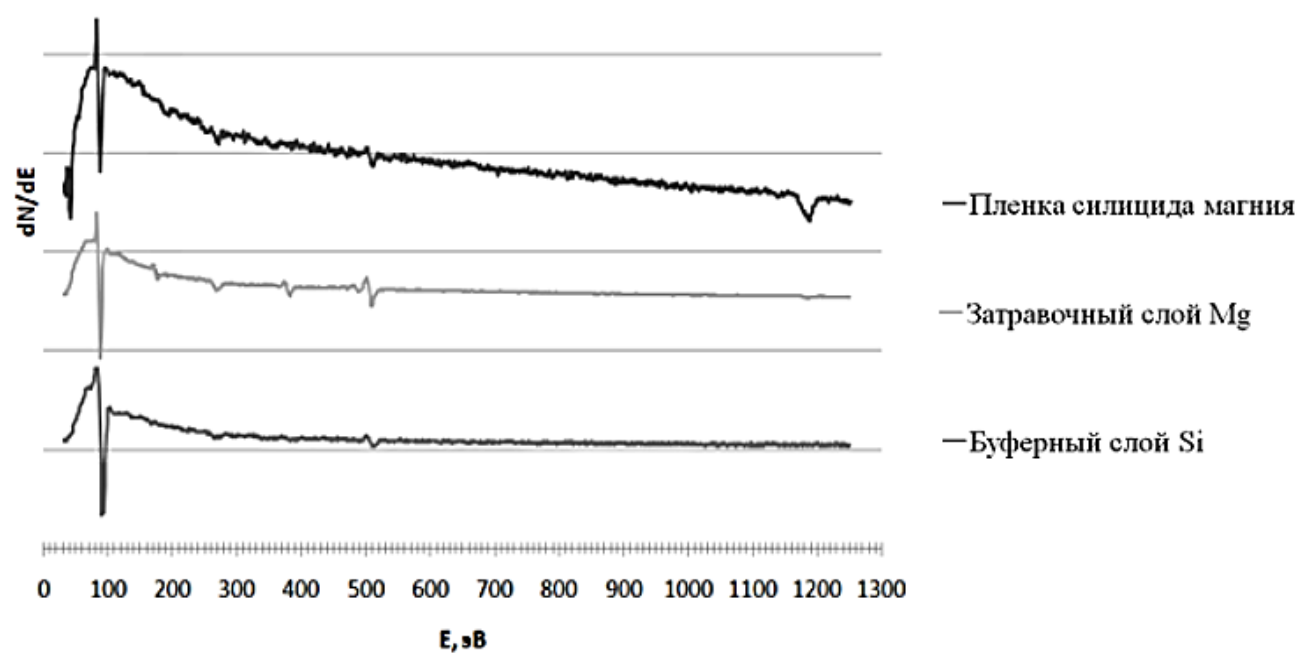

$\mathrm{Si}$, затравочного слоя $\mathrm{Mg}$ и тонкой пленки $\mathrm{Mg}_{2} \mathrm{Si}$.

Puc. 1. Оже-спектры образца, полученные в ходе его формирования.

Анализ спектров оже-электронов показал, что на графике наблюдаются обратные пики, близкие по интенсивности, с энергией 92 эВ, соответствующие по атласу спектров оже-электронов [11] кремнию. На этапе формирования затравочного слоя наблюдается обратный пик малой интенсивности с энергией 1186 эВ, согласно [11] принадлежащий магнию. На этапе формирования тонкой пленки силицида магния в дополнение к указанным пикам наблюдается обратный пик с энергией 45 эВ, по атласу также принадлежащий Mg. Помимо того, на спектрах наблюдаются обратные пики небольшой интенсивности примесей: С (272 эВ) и О (503 эВ).

На рис. 2 приведены спектры ХПЭЭ образца на различных этапах его формирования. На спектре ХПЭЭ буферного слоя $\mathrm{Si}$ отчетливо видны пики с максимумами при энергиях 10 эВ и 17.5 эВ, принадлежащие соответственно поверхностному и объемному плазмонам кремния, что хорошо соотносится со справочными данными [12].

На этапе формирования затравочного слоя $\mathrm{Mg}$ наблюдается уменьшение вклада объемного плазмона $\mathrm{Si}$, а также смещение в сторону меньших энергий пиков, принадлежащих поверхностному и объемному плазмонам, энергии максимумов которых 9 эВ и 16.5 эВ соответственно. Это свидетель- 


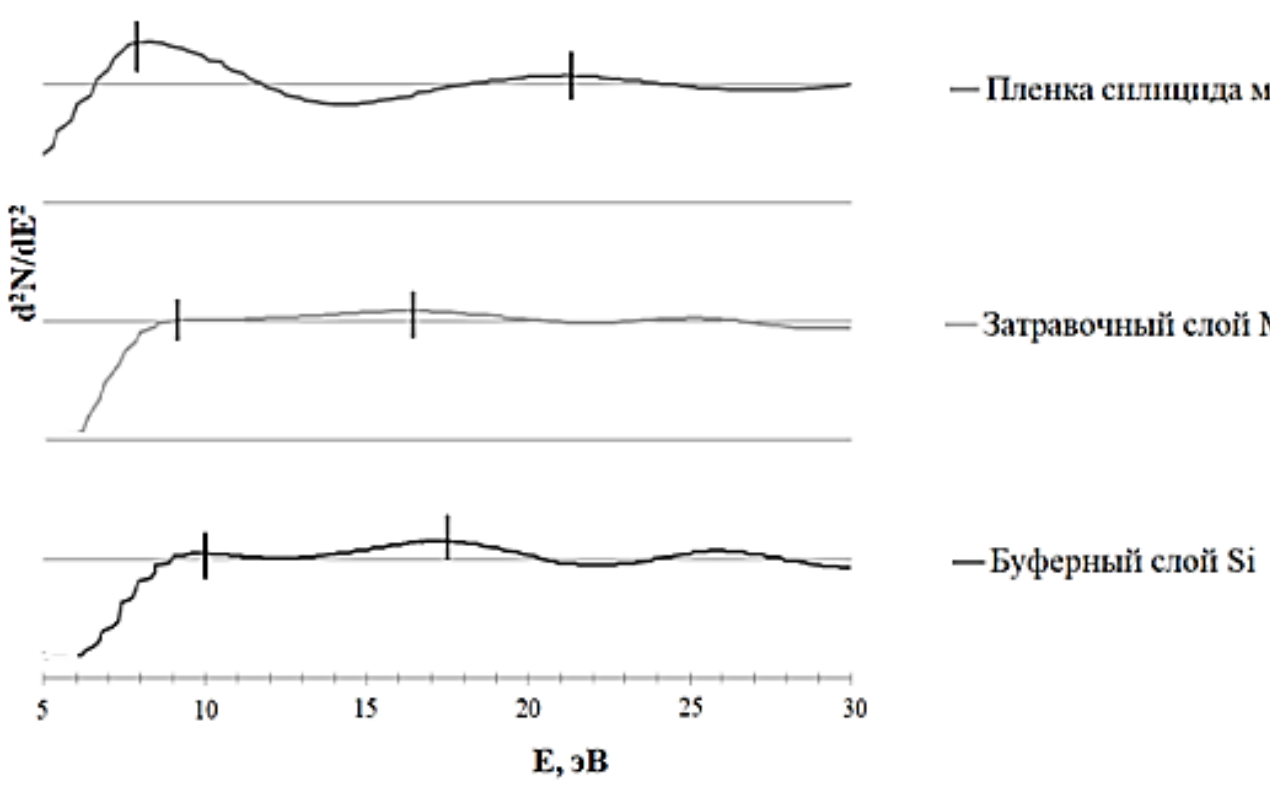

ствует, что на поверхности образца сформирована тонкая несплошная пленка $\mathrm{Mg}$. Ее формирование проходило согласно работе [5] по островковому типу.

Puc. 2. Спектры ХПЭЭ полученные в процессе формирования образца.

После формирования 100 нм пленки силицида магния на соответствующем спектре ХПЭЭ наблюдается интенсивный пик с энергией максимума порядка 7 эВ и пик с большой полушириной при энергиях от 17 эВ до 23 эВ с энергией максимума 21.5 эВ.

Сдвиг в сторону меньших энергий свидетельствует о формировании обогащенного кремнием силицида магния. Это подтверждается и его спектром оже-электронов (рис. 1), на котором интенсивность обратного пика кремния (92 эВ) значительно больше интенсивности обратного пика магния (1186 эВ).

Таким образом, в результате проведенного эксперимента по отработке методики формирования тонких пленок $\mathrm{Mg}_{2} \mathrm{Si}$ методом твердофазной эпитаксии была получена пленка толщиной 100 нм, которая in-situ были исследована методами ЭОС и СХПЭЭ. Анализ спектров оже-электронов показал наличие атомов магния и кремния в составе сформированной пленки. Из анализа спектров ХПЭЭ было установлено, что была сформирована тонкая пленка силицида магния, обогащенного кремнием. Определение типа силицида магния образца будет осуществлено позднее, методом рентгеноструктурного анализа, после чего будет проведена корректировка этапов формирования тонких пленок $\mathrm{Mg}_{2} \mathrm{Si}$.

1. Semiconducting $\mathrm{Mg}_{2} \mathrm{Si}$ thin films prepared by molecular-beam epitaxy/John E. Mahan, Andre' Vantomme, and Guido Langouche //Physical review B. - 1996. - Vol. 54, № 23. - P. 16965-16971.

2. Udono, H., Yamanaka, Y., Uchikoshi, M., Isshiki, M. Infrared photoresponse from pn-junction $\mathrm{Mg}_{2} \mathrm{Si}$ diodes fabricated by thermal diffusion // J. Phys. Chem. Solids. - 74,311-314.

3. Akiyama, T., Hori, N., Tanigawa, S., Tsuya, D., Udono, H. Fabrication of MgSipnjunction photodiode with shallow mesa-structure and ring electrode // 011102 JJAP Conf. Proc. 5. - 2017.

4. Zaitsev, V.K., Fedorov, M.I., Gurieva, E.A., Eremin, I.S., Konstantinov, P.P., Samunin, A.Y., Vedernikov, M.V. Highly effective $\mathrm{Mg}_{2} \mathrm{Si}_{1-\mathrm{x}} \mathrm{Sn}_{\mathrm{x}}$ thermoelectrics // 045207Phys. Rev. B 74. - 2006.

5. Solid phase growth and properties of $\mathrm{Mg}_{2} \mathrm{Si}$ films on $\mathrm{Si}(111)$ / N.G. Galkin, S.V. Vavanova, A.M. Maslov, K.N. Galkin, A.V. Gerasimenko, T.A. Kaidalova // Thin Solid Films 515. - 2007. - P. 8230-8236.

6. Probing the $\mathrm{Mg}_{2} \mathrm{Si} / \mathrm{Si}(111)$ heterojunction for photovoltaic applications /Alexander Shevlyagin, Igor Chernev, NikolayGalkin, AndreyGerasimenko, Anton Gutakovskii, Hirofumi Hoshida, Yoshikazu Terai, Naofumi Nishikawa, Keisuke Ohdaira // Solar Energy-2020. - 211. - 2020. - P. 383-395.

7. A study of the temperature dependence of adsorption and silicidation kinetics at the $\mathrm{Mg} / \mathrm{Si}(111)$ interface / K.N. Galkin, Mahesh Kumar, Govind, S.M. Shivaprasad, V.V. Korobtsov, N.G. Galkin // Thin Solid Films-2007. 515. - Р 8192-8196.

8. Твердофазный рост и структура пленок дисилицида бария на $\operatorname{Si}(111)$ / В.Л. Дубов, Д.В. Фомин, Н.Г. Гал- 
кин // Вестник Самарского гос. аэрокосмического ун-та. - 2016. - Т. 15, № 2.- С. 114-121.

9. $\mathrm{BaSi}_{2}$ - перспективный материал для фотоэлектрических преобразователей / В.Л. Дубов, Д.В. Фомин // Успехи прикладной физики. - 2016. - Т. 4 , № 6. - С.599-605.

10. Взаимосвязь оптических и фотоэлектрических свойств пленок и диодных гетероструктур на основе $\mathrm{BaSi}_{2}$ и $\mathrm{Si}(111)$ / Н.Г. Галкин, Д.Л. Горошко, В.Л. Дубов, Д.В. Фомин, К.Н. Галкин, Е.А. Чусовитин, С.В. Чусовитина // Химическая физика и мезоскопия. - 2019. - Т. 4, № 6. - С. 375-385.

11. Handbookof Augerelectron spectroscopy: A reference book of standard data for identification and interpretation of Auger electron spectroscopy data //Paul William Palmberg. Physical Electronics Industries, 1972 - P. 160.

12. Лифшиц, В.Г., Луняков, Ю.В., Спектры ХПЭЭ поверхностных фаз на кремнии. - Владивосток: Дальнаука, 2004. - 315 с.

УДК 533.6.011

Верхотурова Ирина Владимировна

Амурский государственный университет

г. Благовещенск, Россия,

E-mail: loy-iver@rambler.ru

Verkhoturova Irina Vladimirovna

Amur State University,

Blagoveshchensk, Russia

E-mail: loy-iver@rambler.ru

\section{МОДЕЛИРОВАНИЕ ОБТЕКАНИЯ ТЕЛ С ОТРЫВОМ ПОТОКА ЖИДКОСТИ В СРЕДЕ COMSOL MULTIPHYSICS}

\section{SIMULATION OF BODY FLOW WITH A SEPARATED LIQUID FLOW IN A COMSOL MULTIPHYSICS ENVIRONMENT}

Аннотация. В статье представлены результаты применения среды COMSOL Multiphysics для выполнения одной из лабораторных работ модуля "Аэродинамика» дисциплины «Гидрогазоаэродинамика». В среде COMSOL Multiphysics предлагается провести моделирование процесса обтекания ламинарным потоком вязкой несжимаемой жидкости тел различной геометрической формы, что позволяет наглядно визуализировать пограничный слой, его отрыв от поверхности обтекаемого тела.

Abstract. The article presents the results of using the COMSOL Multiphysics environment to perform one of the laboratory works of the Aerodynamics module of the Hydrogas and Aerodynamics discipline. In the COMSOL Multiphysics environment, it is proposed to simulate the process of a laminar flow of a viscous incompressible fluid around bodies of various geometric shapes, which allows you to visually visualize the boundary layer, its separation from the surface of the streamlined body.

Ключевые слова: модельный эксперимент, лабораторные работы, COMSOL Multiphysics, обтекание тел, ламинарный поток, число Рейнольдса.

Key words: model experiment, laboratory work, COMSOL Multiphysics, flow around bodies, laminar flow, Reynolds number.

DOI: $10.22250 /$ jasu.93.7

В настоящее время компьютерное моделирование как метод исследования различных физических процессов прочно заняло место не только в системе научных исследований, но и в современном лабораторном практикуме. Модельный эксперимент, не являясь альтернативой реальному, с помо- 